Original Research Paper

\title{
Efficient Scheduling of Packets in Wireless Sensor Networks Using Priority Based Scheduling Approach
}

\author{
${ }^{1}$ B. Booba and ${ }^{2}$ T.V. Gopal \\ ${ }^{I}$ Research Scholar, Anna University, Chennai, Tamil Nadu, India \\ ${ }^{2}$ Professor, Department of Computer Science and Engineering, Anna University, Chennai
}

\author{
Article history \\ Received: 01-05-2014 \\ Revised: 03-06-2014 \\ Accepted: 06-08-2014 \\ Corresponding Author: \\ Booba, B \\ Research Scholar, Anna \\ University, Chennai, Tamil Nadu, \\ India \\ Email: boobaphd@gmail.com
}

\begin{abstract}
The packet scheduling is essential and plays an important role to sense the element nodes with resource constraints in Wireless Networks. The packet scheduling scheme also scale the energy consumption and end-to-end information transmission delay in time period and non-real-time information packets during wireless transmission. The proposed Priority based Packet Scheduling (PPS) technique has a tendency to overcome the issues based on priority queues in a wireless network. The priority queue has three levels at each node, except those at the last virtual hierarchy level in the zonebased topology of wireless network. In the meantime packet square are measured and placed in the highest-priority queue and might preempt information packets in alternative queues. In the non-real-time period packet square are measured and placed into two alternative queues supported at a particular threshold of their calculable interval. The time period and non-real time information has two queues in a leaf node, since they did not receive information from alternative nodes which causes scale back end-to-end delay. The proposed PPS technique has affinity to evaluate performance through simulations for the time period and non-real-time information. Simulation results illustrate that the PPS Scheme outperforms typical schemes in terms of average information waiting time and end-to-end delay.
\end{abstract}

Keywords: End-to-End Delay, Non Pre-Emptive, Pre-Emptive, Priority Queue, Data Packets

\section{Introduction}

There are few issues in network designing, such as routing protocols and data aggregation that reduce sensor energy consumption and data transmission delay, packet scheduling at sensor nodes. It seems to be highly important since it ensures delivery of different types of data packets based on their priority with least idleness.

For example, data sensed for progressing orders have higher need than data sensed for non-real-time demands. Regardless of the way that plentiful number of exploration are ruined scheduling the sleep wake times of sensor nodes (Anastasi et al., 2009; Bergmann et al., 2010) simply several studies exist in the composition on the packet scheduling of sensor nodes (Edalat et al., 2009) that schedule the get ready of information packet open at a sensor center point which all the more moreover abatements vitality uses.

Certainly, most existing Wireless Sensor Network (WSN) operating systems utilize First Come First Serve (FCFS) (Stallings, 1995) to process a data packet which thusly devours more of an opportunity to convey them to applicable Base Station (BS). In any case the data packets are relied upon to be conveyed to the BS before the deadline happens. Furthermore constant crisis data must be conveyed first to BS as soon as possible. Henceforth the hubs must have the capacity to change the conveyance request of packets based on their necessities. 
Moreover, most existing packet A algorithms of WSN are not dynamic or suitable for far reaching scale procurements taking after these schedulers are fated and static and can't be changed on account of a change in the procurement requirements of course situations (Zhao et al., 2009).

Keeping in mind the end goal to defeat these troubles Priority based Packet Scheduling (PPS) plan is proposed in this study. The sensor hubs take after a various levelled structure which is practically sorted out. The pecking order is chosen based on the jump separation of the hubs in Bs. TDMA plan is utilized to process the information sensed by hubs at diverse levels. Every hub keeps up three level of priority queue (Stallings, 1995).

The rest of the study is organized as follows. In Section II, we will discuss about related works. Section III presents problem statement. Section IV provides details about assumption and terminologies used in Priority based Packet Scheduling (PPS). Section V evaluates the performance. Section VI is about performance analysis. Finally, Section VII concludes the study.

\section{Related Works}

In this segment, we will be talking about existing packet scheduling schemes by arranging them dependent upon a few factors, for example, deadline, priority, packet sort, number of queue. Notwithstanding we can talk about them in subtle element. Taking into account the deadline of entry time of data to the BS the scheduling schemes could be arranged which are as takes after.

First Come First Serve (FCFS): Most of the existing WSN provisions use FCFS schedulers which forms the data in the request of their arrival times. In FCFS, the data that arrive late at the middle nodes of the system from the far off leaf nodes needs a lot of time to be conveyed to BS yet data from close by neighbouring nodes take less time to be handled at the halfway nodes. In view of which they encounter long waiting times.

Earliest Deadline First (EDF): When vast number of data packets are accessible at the queue with deadlines, inside which it ought to be sent to BS, the data packet which has the earliest deadline is sent first. This algorithm is distinguished to be profitable the extent that normal packet waiting time and end-toend delay (Lu et al., 2002).

In view of the priority of packets sensed at diverse nodes the scheduling plan might be arranged. Think about two packets $\mathrm{p} 1$ and $\mathrm{p} 2$. Non-preemptive: In nonpreemptive priority packet scheduling, exactly when a packet p1 starts execution, undertaking $p 1$ is completed regardless of the fact that the packet $\mathrm{p} 2$ has higher priority than the present running packet $\mathrm{p} 1$ arrives great to go queue. Hence p2 need to hold up in the arranged queue until the execution of $\mathrm{p} 1$ is done.

Preemptive: In preemptive priority packet scheduling, higher priority packets are handled first by sparing the connection of easier priority packets in the event that they are as of now running (Stallings, 1995). $\mathrm{Yu}$ et al. (2008). In view of the packet sort the scheduling plan is as takes after. Continuous packet scheduling: Packets are no doubt scheduled dependent upon their sorts and priorities. The most noteworthy priority around all information packets goes to the Real-time information packets in the primed queue. Thus, they are prepared first and conveyed to the BS with a base end-to-end delay. Non-real time packet scheduling: Non-ongoing packets have more level priority than constant undertakings. They are conveyed to BS either utilizing FCFS or SJF foundation when there is no continuous packet. These packets might be naturally appropriated by ongoing packets (Zhao et al., 2009). Momeni et al. (2009) proposed a two-level task allocation technique that first breaks down the end-toend tasks into real time tasks and utilizes suitable algorithm for sensing and acting tasks.

In view of the amount of levels in the ready queue of a sensor hub the scheduling plan is as takes after. Single Queue: Each and every sensor hub has one ready queue. Various types of information packets enter the ready queue and are scheduled reliant upon differing criteria: Sort, need, size, et cetera. Single queue scheduling has a high famishment rate. Multi-level Queue: Each hub has more than one queue. Information packets are situated into the unique queues agreeing to their priorities and sorts. In this way, scheduling has two phases: (i) Assigning tasks around distinctive queues, (ii) scheduling packets in each one queue. The amount of queues at a hub relies on upon the level of the hub in the network (Zhao et al., 2009; Lee et al., 2010).

Okhovvat and Sharifi (2011) proposed a two phase allocation technique that is based on queuing theory. Tasks are equally distributed to actors in the first phase to estimate the capacity of each actor in order to perform the allocated tasks. The tasks are the assigned to actors based on their estimated capacity so that it reduces the total completion time of the tasks in the entire network.

\section{Preliminaries}

The task scheduling arrangement are masterminded dependent upon particular factors like priority, packet size, time delay and so on., Thus in this study, we are proposing a Priority based Packet Scheduling (PPS) 
plan for Wireless Sensor Networks (WSNs). This framework uses three-level of priority queues to schedule data packets dependent upon their sort and necessities. It ensures end-to-end data transmission for the most important priority data while demonstrating satisfactory fairness towards minimum priority data. Experimental results demonstrate that the proposed PPS packet scheduling arrangement has favoured execution over the existing FCFS and Multilevel Queue Scheduler the extent that the typical assignment holding up time and end-to-end delays (Nasser et al., 2013).

\section{Dynamic Multilevel Priority Packet Scheduling Scheme}

This section is about the Priority based Packet Scheduling (PPS) scheme. It is an scheduling algorithm which calculates the priorities of packets in the course of the system execution. The objective of PPS is to adjust to dynamically changing progress and should form an optimal solution in self-sustained manner. It might be hard to produce definite policies to achieve the goal depending on the strain of a given problem. Few examples of PPS are earliest deadline first scheduling and Least slack time scheduling (Hwang et al., 2010).

\section{A. Pseudo Code for Proposed Algorithm}

While task ${ }_{c},{ }_{v}$ is received by node ${ }_{v}$ at level $c$, i.e., $x_{c}$ do If type $\left(\operatorname{task}_{\mathrm{c}, \mathrm{v}}\right)=$ real -time then placetask $_{c, v}$ into $\mathrm{pr}_{1}$ queue else if node $\mathrm{v}$ is not at lowest levels then if $\operatorname{task}_{\mathrm{c}, \mathrm{v}}$ is not local then placetask $\mathrm{c}_{\mathrm{c}, \mathrm{v}}$ into $\mathrm{pr}_{2}$ queue else end if placetask ${ }_{c, v}$ into $\mathrm{pr}_{3}$ queue

else

placetask ${ }_{c, v}$ into $\mathrm{pr}_{2}$ queue

end if

Assume, the duration of a timeslot at $x_{c} \leftarrow y(c)$

Data sensing time of $\operatorname{node}_{v}$ at $x_{c} \leftarrow \operatorname{senseTime}_{c}(y)$

Remaining time after data sensing, $y_{1}(c)=y(c)-$ senseTime $_{c}(y)$

Let total real-time tasks for node $v_{v}$ at $x_{c} \leftarrow N_{c}\left(p r_{1}\right)$

Let:

$$
\operatorname{procTimepr}_{1}(c) \leftarrow \sum_{k=0}^{N e(p r 2)} \operatorname{procTime}(k)
$$

if procTime $p r_{1}(c)<y_{1}(c)$ then
All $\mathrm{pr}_{1}$ tasks of node ${ }_{\mathrm{v}}$ at $\mathrm{x}_{\mathrm{c}}$ are processed as FCFS Remaining time $y_{2}(c) \leftarrow y 1(c)-$ procTime $p r_{1}(c)$ Let, total $p r_{2}$ tasks for node ${ }_{v}$ at $x_{c} \leftarrow N_{c}\left(p r_{2}\right)$

Let:

$$
\operatorname{procTimepr}_{2}(c) \leftarrow \sum_{k=1}^{N e(p r 2)} \operatorname{procTime}(k)
$$

if $\operatorname{procTimepr}_{2}(c)<y_{2}(c)$ then

All $p r_{2}$ tasks are processed as FCFS

$\mathrm{pr}_{3}$ tasks are processed as FCFS for the else remaining time, $y_{3}(c) \leftarrow y_{2}(c)$-procTimepr $2(c)$

$\mathrm{pr}_{2}$ tasks are processed for $y_{2}(y)$ time no $p r_{3}$ tasks are processed

end if

else

only $p r_{1}$ tasks are processed for $y_{1}(c)$ time no $p r_{2}$ and $p r_{3}$ tasks are processed end if if $p r_{1}$ queue empty and $p r_{2}$ tasks are processed $\beta$ consecutive imeslots since $y(c) \leq$ procTime $p r_{2}(c)$ then $\mathrm{pr}_{2}$ tasks are preempted at $\beta+1, \ldots, \beta+k$ timeslots by $p r_{3}$ tasks

if $p r_{1}$ task arrives during any of $\beta+1, \beta+2, \ldots, \beta$ $+k$ timeslots then

$p r_{3}$ tasks are resumed and $p r_{1}$ tasks are functioned context are transferred to change again for functioning $p r_{3}$ tasks end if

end if

end while.

\section{Performance Evaluation}

Transmission time or delay that is required to place a real-time data from a node into the medium is equal to data ${ }_{p t r} / s_{t}$. The propagation time or delay to transmit data from the source to destination can be formulated as $d / s_{p}$, considering the above mentioned scenario the end-to-end delay for sending a real-time data satisfies the following inequality Equation 1:

$$
\begin{aligned}
& \text { Delay }_{p r 1}>=x_{c} *\left(\left(\text { data }_{p r 1} / S_{t}\right)+\left(p r 1_{p r o c}(y)\right)\right) \\
& +\left(d / S_{p}\right)+\left(x_{c} * \text { yoverhead }\right)
\end{aligned}
$$

where, datapr 1 denotes the real-time data size, $s_{t}$ denotes the data transmission speed, $d$ is the distance from the source node to $\mathrm{BS}, \mathrm{pr}_{1}$ proc $(y)$ is the processing time of real-time tasks at each node and $\mathrm{y}_{\text {overhead }}$ is an overhead in terms of context switching and queuing time. 
Therefore, the end-to-end delay for a real-time task $y 1$ considering that $y 1$ has $N_{p r 1}$ number of real-time tasks ahead of it Equation 2:

$$
\text { Delay }_{y 1}>=\sum_{j=1}^{N p r 1}(\text { delaypr } 1)
$$

The $p r 2$ tasks are processed in the remaining time of the timeslots. The transmission time or delay to place $p r 2$ data from a node into the medium can be therefore computed as data $\operatorname{drr}_{2} / s_{t}$. Thus, the total endto-end delay for a $p r 2$ task that can be processed in the same timeslot exceeds Equation 3:

$$
\begin{aligned}
& x_{c} *\left(\left(\text { data }_{p r 1} / s_{t}\right)+\left(p r 1_{\text {proc }}(y)\right)\right)+\left(d / s_{p}\right) \\
& +\left(x_{c} * y_{\text {overhead }}\right)
\end{aligned}
$$

The transmission time or delay to place $p r 3$ data from a node into the wireless medium is equal to data $_{p r 3} / s_{t}$. However, during the processing of the $p r 3$ queue tasks, these tasks can be preempted by real-time tasks. They are processed again after the completion of real-time tasks. Thus, the end-to-end delay for processing $p r 3$ tasks will be exceeding Equation 4:

$$
\begin{aligned}
& \beta^{*} y(c)+x_{c} *\left(\left(\text { data }_{p r^{3}} / s_{t}\right)+\left(p r 3_{p r o c}(y)\right)\right) \\
& +\left(d / s_{p}\right)+\left(x_{c} * \text { yoverhead }\right)
\end{aligned}
$$

Since $p r 1$ tasks are processed as FCFS, the average waiting time for real-time, $p r 1$ tasks at node $v$ is Equation 5:

$$
A W \operatorname{Tpr} 1(y)=\sum_{k 1=1}^{N 1-1} \sum_{a=1}^{k 1} p r 1, a(y) / N 1
$$

If $p r 2$ tasks are not preempted by $p r 1$ tasks and can be completed within the $y 2(c)$ time (i.e., within the same timeslot for the processing $p r 1$ tasks), the average waiting time for $p r 2$ tasks can be expressed as follows Equation 6:

$$
A W T p r 2(y)=\sum_{k 2=1}^{N 2-1} \sum_{a=1}^{k 2} p r 2, a(y) / N 2
$$

\section{Performance Analysis}

Simulation model is employed to judge the performance of the proposed PPS scheme, scrutiny it against the FCFS and construction Queue planning schemes. The comparison is created in terms of average packet waiting time and end-to-end knowledge transmission delay. These systems tend to use willy-nilly connected Unit Disk Graphs (UDGs) on a surface of one hundred meter one hundred meter as a basis of our simulations. The amount of simulated zones varies from four to twelve zones. Nodes are distributed uniformly over the zones. The prepared queue of every node will hold a most of fifty tasks. Every task includes a kind ID that identifies its kind. For example, kind zero is taken into account to be a period of time task. Knowledge packets are placed into the prepared queue supported the interval of the task. Moreover, every packet includes a hop count range that's assigned willy-nilly and therefore the packet with the very best hop count range is placed into the highest-priority queue. This system tends to run the simulation each for a selected range of zones and levels within the network till knowledge from a node in every zone or level reach baccalaureate. Simulation results are conferred for each period of time knowledge and every one sorts of knowledge traffic. Table 1 shows comparison of the proposed approach with other scheduling approaches. The results show that the proposed approach outperforms other scheduling algorithms.

From the above simulation results we can conclude that the priority based scheduling method provides high efficient system. Figure 1 system provides the average delay between each packet delivery and this will be useful to calculate the waiting time for each packet Fig. 2 shows the energy consumption of the system during the transmission. Figure 3 represents the throughput of the system. By using priority based scheduling, waiting time of each packet will be reduced based on their priority. So the throughput will always be high for this system. Figure 4 shows the packet delivery ratio of the system. It indicates number of successful delivery of packets to the destination. Figure 5 represents the packet drop during the interruption in the system.

Table 1. Performance comparison

\begin{tabular}{lll}
\hline Algorithm metrics & Priority based scheduling & Other scheduling algorithm \\
\hline Throughput (Mbps) & 246.667 & 292.1750 \\
Packet loss (\%) & 0.43000 & 0.722000 \\
Average delay (ms) & 0.03100 & 0.065000 \\
Energy consumption & Low & Medium \\
\hline
\end{tabular}




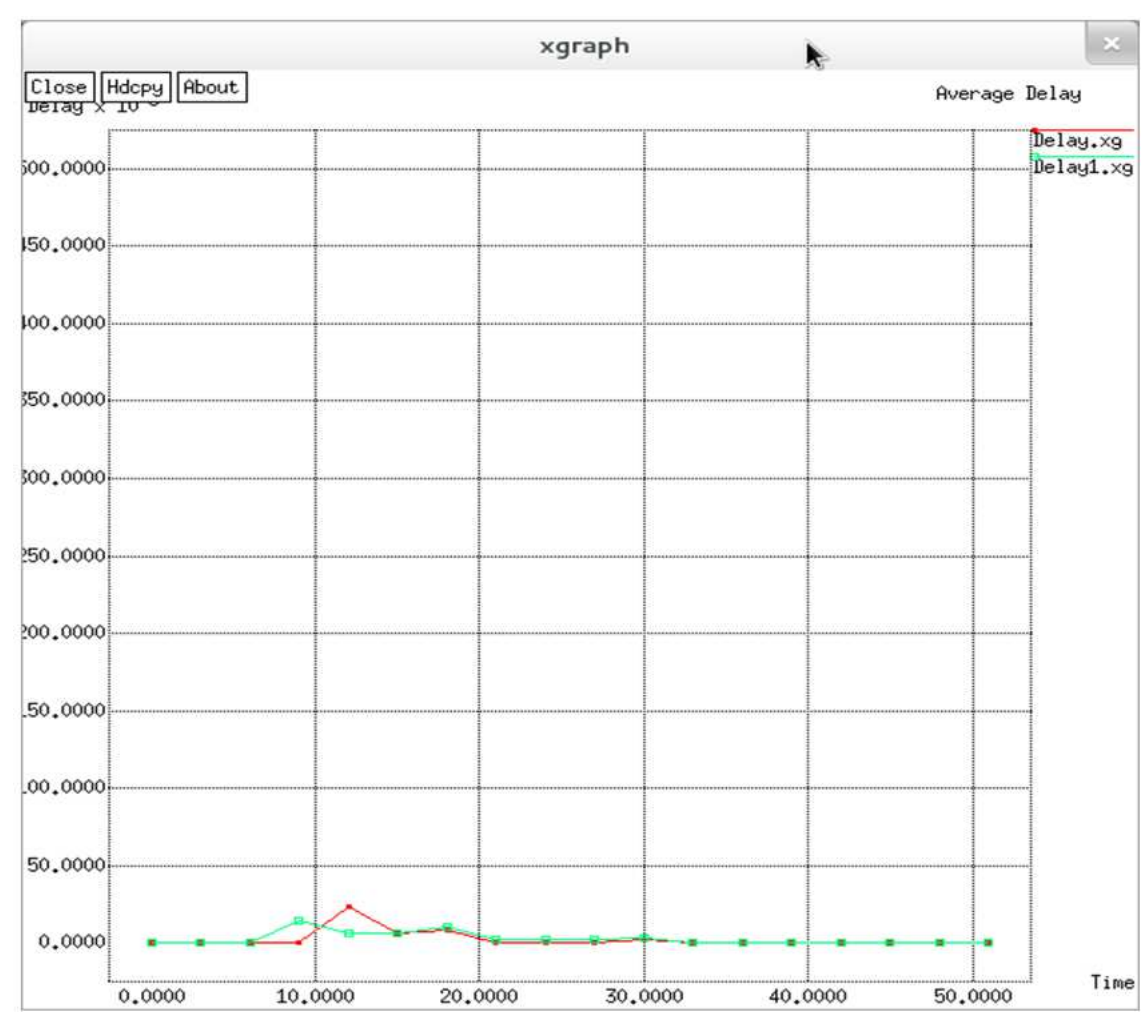

Fig. 1. Average delay during packet transmission

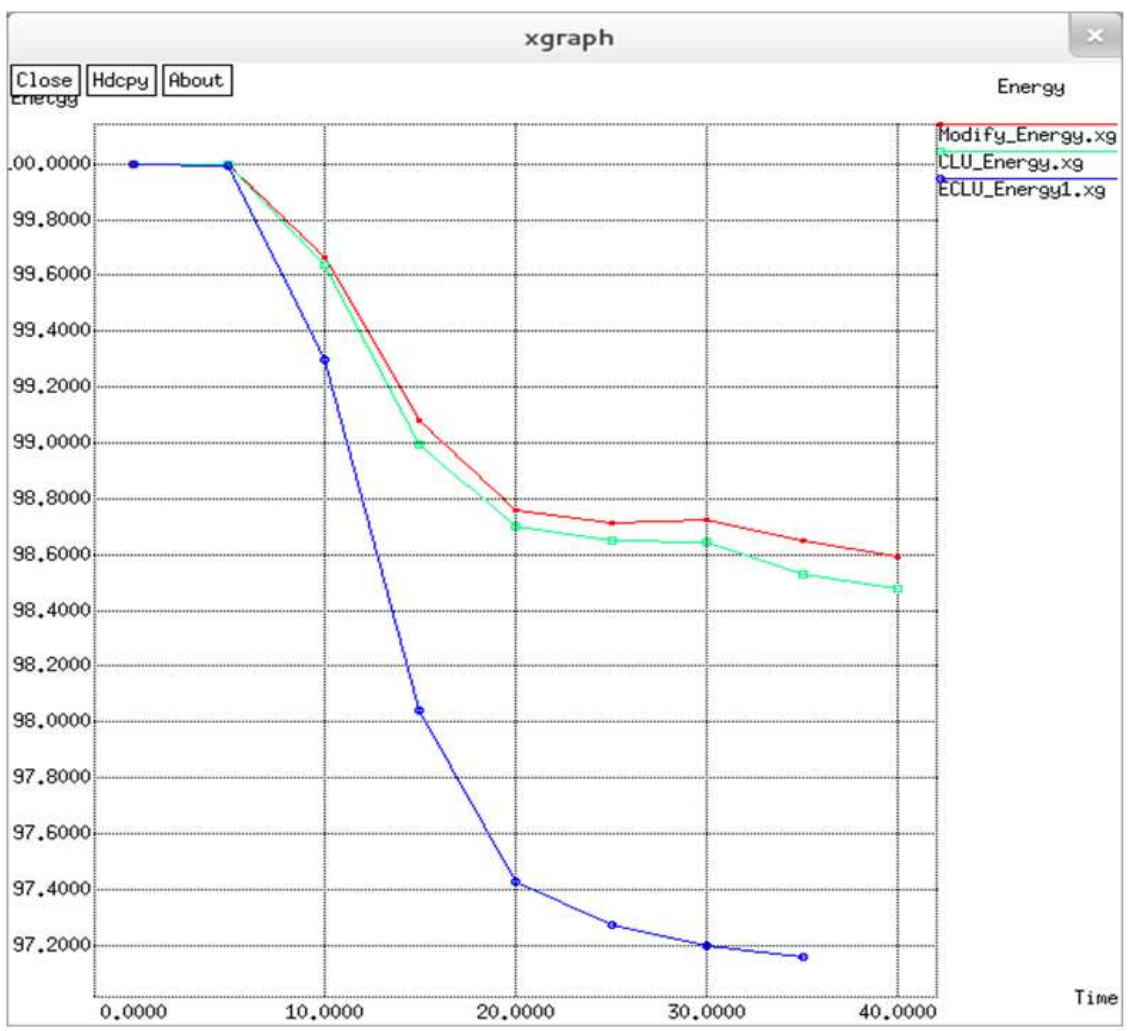

Fig. 2. Energy consumption 


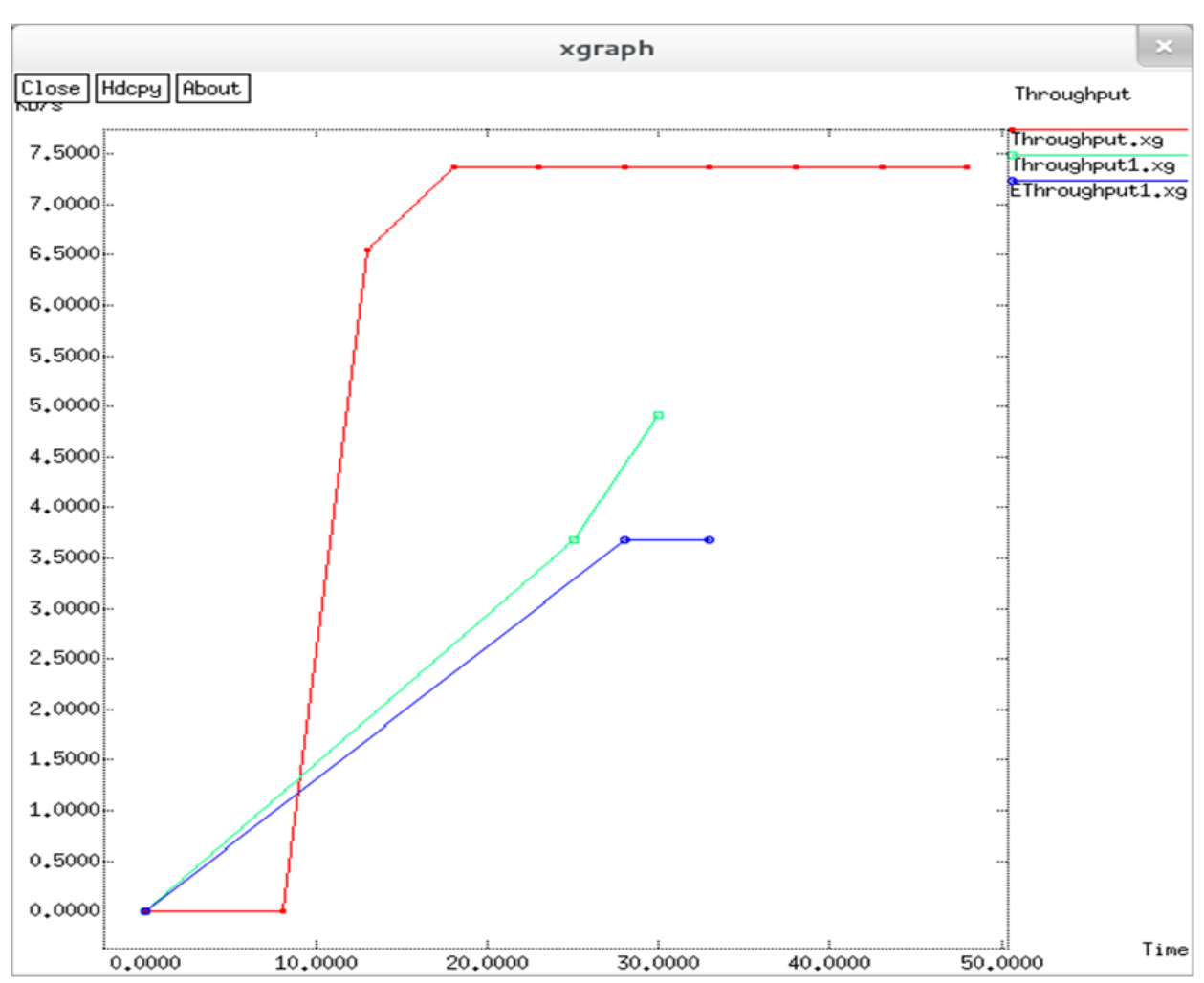

Fig. 3. Throughput

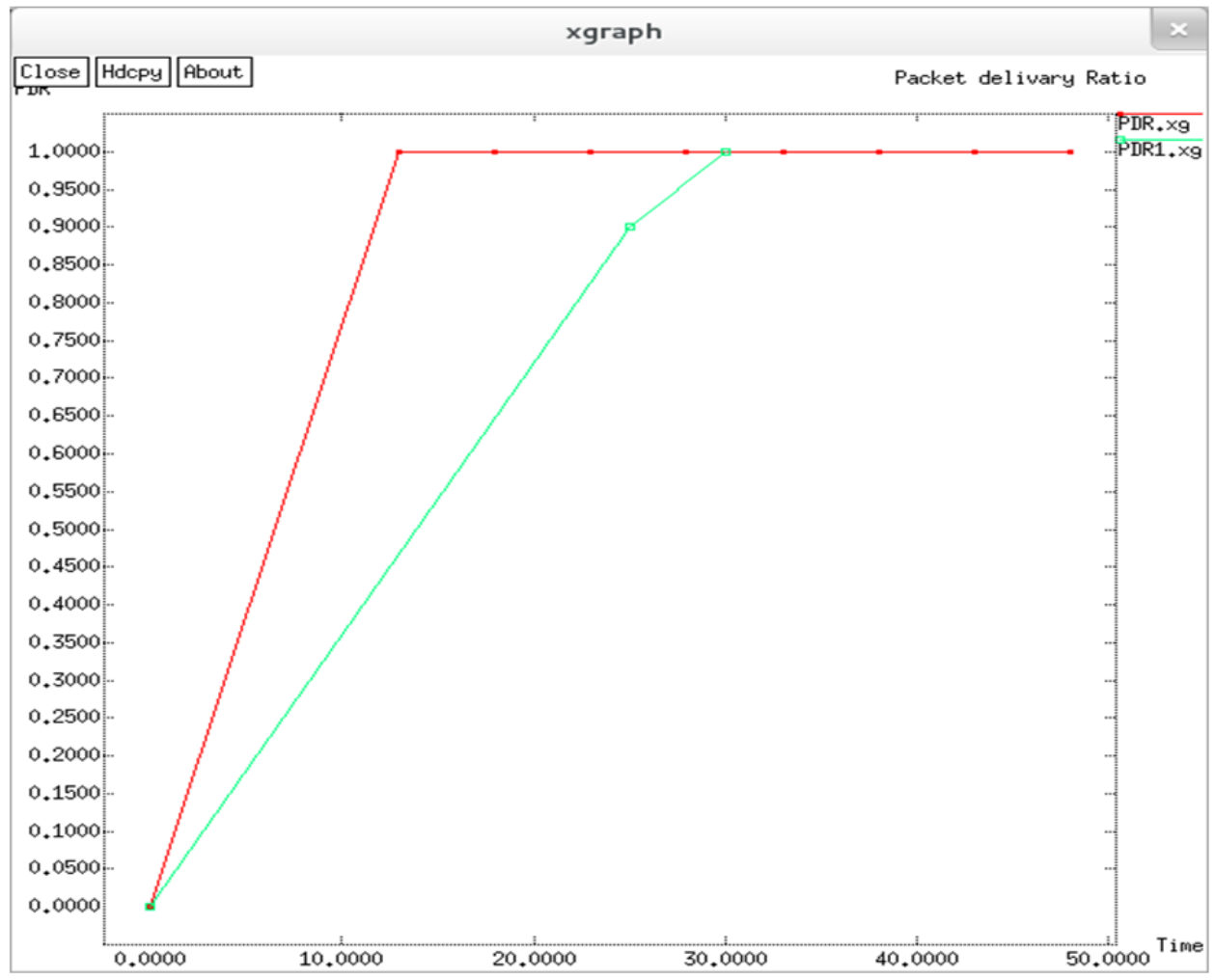

Fig. 4. Packet delivery ratio 


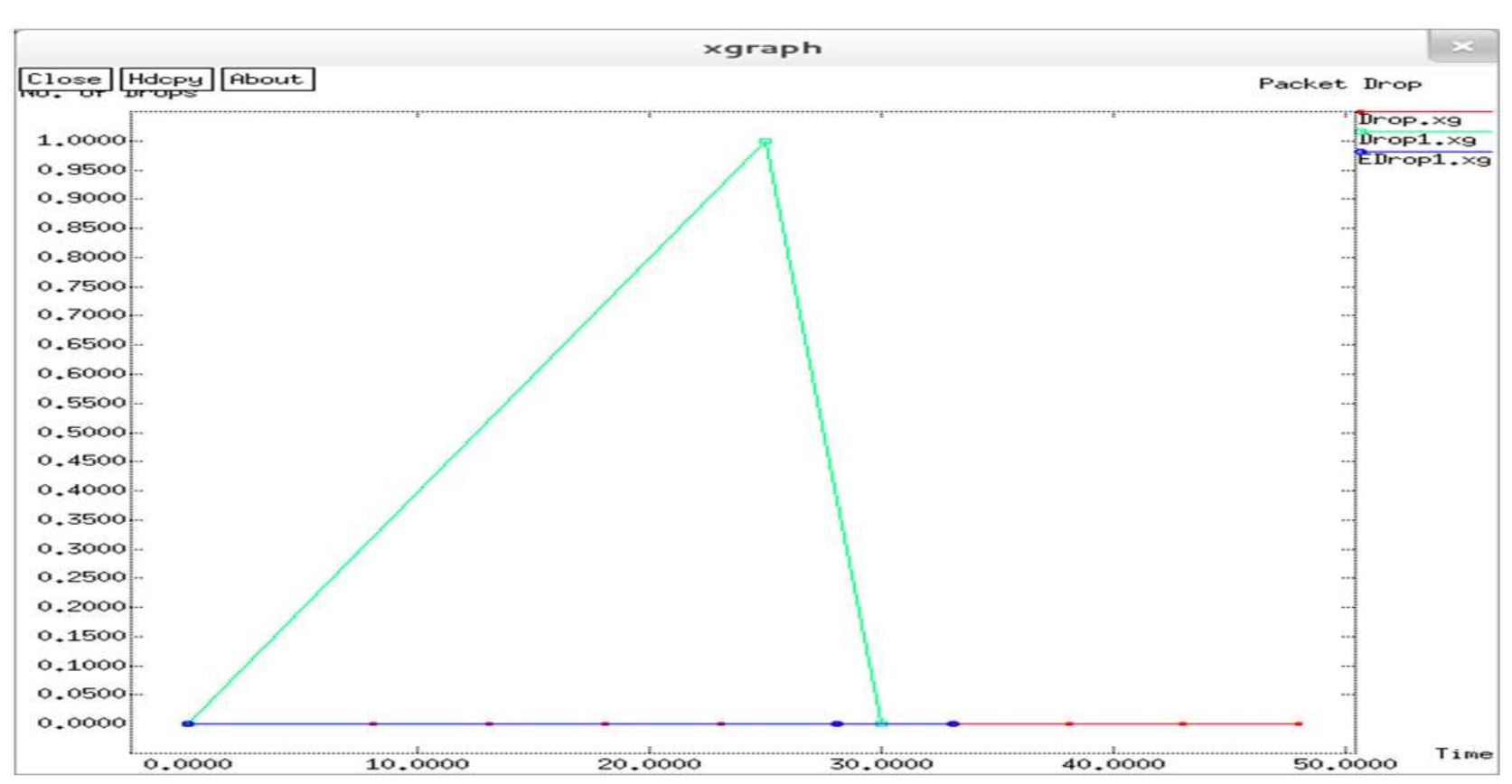

Fig. 5. Packet drop

\section{Conclusion}

In this study, this framework tends to propose a Priority based packet scheduling procedure for Wireless Sensor Networks (WSNs). This system utilizes three-level of priority queues to schedule learning packets dependent upon their assortments and priorities. It guarantees least end-to-end delay transmission for the precise best priority packet in as much as displaying satisfactory equitability towards most reduced priority packet. The anticipated algorithmic rule guarantees an exchange off between priority and reasonableness. Moreover gives higher normal task waiting time and end-to-end delay. Thus, the anticipated PPS task scheduling method is acknowledged to a great degree sparing. However, if a tack holds the resource for a longer time, other tasks waits for a longer time, resulting in deadlock. As enhancement to the proposed approach, pre-emptive scheduling and circular wait will be used to avoid deadlock.

\section{Acknowledgment}

I would like acknowledge my professor and the institution for all the guidance and the support given to me to complete this study.

\section{Author's Contributions}

All authors equally contributed in this work.

\section{Ethics}

This article is original and contains unpublished material. The corresponding author confirms that all of the other authors have read and approved the manuscript and no ethical issues involved.

\section{References}

Anastasi, G., M. Conti and M. Di Francesco, 2009. Extending the lifetime of wireless sensor networks through adaptive sleep. IEEE Trans. Indus. Inform., 5: 351-365. DOI: 10.1109/TII.2009.2025863

Bergmann, G., M. Molnar, L. Gonczy and B. Cousin, 2010. Optimal period length for the CQS sensor network scheduling algorithm. Proceedings of the International Conference Network Services, (CNS' 10), pp: 192-199.

Edalat, N., W. Xiao, C.K. Tham, E. Keikha and L. Ong, 2009. A price-based adaptive task allocation for wireless sensor network. Proceedings of the IEEE International Conference Mobile Adhoc Sensor System, Oct. 12-15, IEEE Xplore Press, Macau, pp: 888-893. DOI: 10.1109/MOBHOC.2009.5337039

Lee, E.M., A. Kashif, D.H. Lee, I.T. Kim and M.S. Park, 2010. Location based multi-queue scheduler in wireless sensor network. Proceedings of the International Conference Advanced Communication Technology, Feb. 7-10, IEEE Xplore Press, Phoenix Park, pp: 551-555. 
Lu, C., B.M. Blum, T.F. Abdelzaher, J.A. Stankovic and T. He, 2002. RAP: A real-time communication architecture for large-scale wireless sensor networks. Proceedings of the IEEE Real-Time Embedded Technology Application Symposium, (AS' 02), IEEE Xplore Press. DOI: 10.1109/RTTAS.2002.1137381

Momeni, H., M. Sharifi and S. Sedighian, 2009. A new approach to task allocation in wireless sensor actor networks. Proceedings of the International Conference Computational Intelligence, Communication System Network, Jul. 23-25, IEEE Xplore Press, ndore, pp: 73-78. DOI: 10.1109/CICSYN.2009.51

Hwang, M.G., D.J. Choi and P.K. Kim, 2010. Least slack time rate first: New scheduling algorithm for multi-processor environment, Feb. 15-18, IEEE Xplore Press, Krakow, pp: 806-811.

DOI: 10.1109/CISIS.2010.20

Okhovvat, M. and M. Sharifi, 2011. Task allocation to actors in wireless sensor actor networks: An energy and time aware technique. World Conf. Inform. Tech., 3: 484-490. DOI: 10.1016/j.procs.2010.12.082
Nasser, N., L. Karim and T. Taleb, 2013. Dynamic multilevel priority packet scheduling scheme for wireless sensor network. IEEE Trans. Wireless Communi., 12: 1448-1459. DOI: 10.1109/TWC.2013.021213.111410

Stallings, W., 1995. Operating Systems. 2nd Edn., Prentice Hall.

Yu, M., S.J. Xiahou and X.Y. Li, 2008. A survey of studying on task scheduling mechanism for TinyOS. Proceedings of the International Conference Wireless Communication Network Mobile Computing, Oct. 12-14, IEEE Xplore Press, Dalian, pp: 1-4. DOI: 10.1109/WiCom.2008.960

Zhao, Y., Q. Wang, W. Wang, D. Jiang and Y. Liu, 2009. Research on the priority-based soft real-time task scheduling in TinyOS. Proceedings of the International Conference Information Technology Computer Science, Jul. 25-26, IEEE Xplore Press, Kiev, pp: 562-565. DOI: 10.1109/ITCS.2009.122 